\section{A definição de medicamentos prioritários para o monitoramento da qualidade laboratorial no Brasil: articulação entre a vigilância sanitária e a Política Nacional de Medicamentos}

\author{
Definition of priority medicines for monitoring \\ laboratory quality in Brazil: the interface between \\ health surveillance and the National Drug Policy
}

${ }^{1}$ Escola Nacional de Saúde Pública Sergio Arouca, Fundação Oswaldo Cruz, Rio de Janeiro, Brasil. 2 Instituto Nacional de Controle de Qualidade em Saúde, Fundação Oswaldo Cruz, Rio de Janeiro, Brasil.

Correspondência D. M. Pontes Junior Departamento de Administração e Planejamento em Saúde, Escola Nacional de Saúde Pública Sergio Arouca, Fundação Oswaldo Cruz. Rua Leopoldo Bulhões 1480, Rio de Janeiro, $R J$ 21041-210, Brasil. durvalmp@yahoo.com.br

\begin{abstract}
A key objective of the Brazilian National Drug Policy is the quality of medicines supplied to the population. This study aimed to set priorities for the analysis of the National Program for Quality Control of Medicines. The main criterion was the drug's presence in at least three Pharmaceutical Care Programs under the Ministry of Health. Additional criteria were presence on the National List of Essential Drugs (RENAME) in 2002 and its indication for the 20 main causes of disabilityadjusted life years (DALY). The sources were data from the Ministry of Health and related legislation. The drugs were classified according to the Anatomical Therapeutic Chemical Classification System (ATC) of the WHO. The 13 pharmaceutical care programs included 893 products classified in 449 different ATC codes. Twenty-eight drugs were considered priorities, 26 of which were listed on the RENAME and 12 indicated as causes of DALY. It is recommended that the National Health Surveillance Agency and Secretariat of Science, Technology, and Strategic Inputs establish an integrated strategy to guarantee comprehensive quality of these drugs, including laboratory quality, registration, good manufacturing practices, and information for health professionals and the general population.
\end{abstract}

Drug Quality; National Drug Policy; Health Surveillance

\author{
Durval Martins Pontes Junior 1 \\ Vera Lúcia Edais Pepe 1 \\ Claudia Garcia Serpa Osorio-de-Castro ${ }^{1}$ \\ Elisa Prestes Massena ${ }^{1}$ \\ Margareth Crisóstomo Portela 1 \\ Maria do Carmo Miranda 2 \\ Raulino Sabino da Silva 1
}

\section{Introdução}

O processo saúde-doença da população é complexo e multicausal. Não obstante, dados recentes são capazes de orientar sobre a importância de algumas doenças, sob o ponto de vista da morbimortalidade e, mais especificamente, da carga a elas atribuída, de anos de vida perdidos por morte prematura ou incapacitação na população brasileira 1,2,3

O estudo - Estimativa da Carga de Doença no Brasil (GBD) - utilizou o indicador DALY (Disability Adjusted Life Years) para medir, simultaneamente, o impacto da mortalidade e dos problemas de saúde que afetam a qualidade de vida dos indivíduos. Estão entre as vinte principais causas a diabetes mellitus, a doença isquêmica do coração, as doenças cerebrovasculares, o transtorno depressivo recorrente/episódio depressivo, a asfixia/traumatismo ao nascer, a doença pulmonar obstrutiva crônica, as infecções de vias aéreas inferiores e doenças degenerativas do sistema nervoso central 1 .

Os medicamentos constituem-se em importantes instrumentos de saúde, que visam minorar o sofrimento, interromper o processo de adoecimento, nos casos de doenças agudas e remissíveis, e melhorar a qualidade de vida dos indivíduos quando portadores de doenças crônicas, retardando seus efeitos maléficos. Instituída no ano de 1998, a Política Nacional de Medicamentos 4 (p. 9) objetiva “... garantir a necessária 
segurança, eficácia e qualidade dos medicamentos, a promoção do uso racional e o acesso da população àqueles considerados essenciais...". Três eixos organizam a ação governamental: (1) a regulação sanitária, que tem por objetivo proteger o consumidor de produtos com qualidade e eficácia duvidosas; (2) a regulação econômica, que visa proteger o consumidor de eventuais abusos praticados pelo mercado; e (3) a assistência farmacêutica, que utiliza ações específicas como pesquisa, desenvolvimento e produção de medicamentos e insumos para promover não apenas o acesso a medicamentos ditos como essenciais, mas também seu uso racional na promoção, proteção e recuperação da saúde 5,6.

Constam da Política Nacional de Medicamentos oito diretrizes que têm, como pedra fundamental, a Relação Nacional de Medicamentos Essenciais (RENAME). Buscam não apenas ações de reorientação da Assistência Farmacêutica no país, mas também outras ações voltadas à regulação sanitária, ao monitoramento da qualidade dos medicamentos, ao desenvolvimento e capacitação de recursos humanos, ao desenvolvimento científico e tecnológico, à produção pública e ao uso racional dos medicamentos. Essas diretrizes agregam quatro prioridades que “... configuram as bases para o alcance do propósito desta Política, bem como para a implementação das diferentes ações indispensáveis ao seu efetivo cumprimento" ${ }^{4}$ (p. 21). São elas: (1) revisão permanente da RENAME; (2) assistência farmacêutica; (3) promoção do uso racional de medicamentos; e (4) organização das atividades de vigilância sanitária de medicamentos.

Consonante com a Política Nacional de Medicamentos, o Ministério da Saúde vem implementando, ao longo dos últimos anos, diferentes Programas de Assistência Farmacêutica, com o objetivo de melhorar o acesso da população brasileira aos medicamentos ofertados pelo Sistema Único de Saúde (SUS) 7, muito embora sem corresponder, necessariamente, a maior eqüidade no acesso. Messeder et al. 8 destacam o aumento de mandados judiciais, contra o Estado do Rio de Janeiro, no período de 1991 a 2001, para o fornecimento de medicamentos que deveriam ser obtidos através de Programas de Assistência Farmacêutica do Ministério da Saúde (Programa Nacional de DST e AIDS, Programa de Medicamentos Excepcionais e Programa de Assistência Farmacêutica na Atenção Básica). Alguns estudos têm também demonstrado que a qualidade dos medicamentos ofertados no país tem sido, historicamente, questionável 9,10,11.

No intuito de contribuir para o monitoramento da qualidade de medicamentos, a Agência Nacional de Vigilância Sanitária (ANVISA), em conjunto com o Instituto Nacional de Controle de Qualidade em Saúde, Fundação Oswaldo Cruz (INCQS/FIOCRUZ), instituiu o Programa Nacional de Verificação da Qualidade de Medicamentos (PROVEME). Esse tem como objetivo geral implantar um programa nacional de análise laboratorial de medicamentos, visando monitorar a qualidade desses produtos, para promover o saneamento do mercado e para atuar de forma a construir um modelo de intervenção preventiva, ou seja, antes de haver dano ou agravo à saúde da população 12. A sua implementação, bem como a sua continuidade e o seu aprimoramento configuram-se como um grande desafio e necessitam estar articulados à Política Nacional de Medicamento e a uma lógica de saúde pública que considere a morbimortalidade da população brasileira.

Este trabalho objetiva definir medicamentos considerados prioritários para a ação do PROVEME, considerando os distintos Programas de Assistência Farmacêutica do Ministério da Saúde, a RENAME e os medicamentos que fazem parte do esquema terapêutico das principais causas de DALY no Brasil.

\section{Métodos}

No sentido de atingir o objetivo deste trabalho, três etapas foram realizadas: (1) a identificação dos programas de assistência farmacêutica do Ministério da Saúde; (2) a construção de um banco de dados contendo todos os medicamentos e produtos não terapêuticos encontrados nos programas de assistências farmacêuticas e na RENAME; e (3) a definição dos medicamentos considerados prioritários para a ação do PROVEME, propriamente.

\section{Identificação dos programas de assistência farmacêutica do Ministério da Saúde}

Para identificar os Programas de Assistência Farmacêutica e seus respectivos elencos de medicamentos, realizou-se um levantamento bibliográfico, na base de dados da Biblioteca Virtual em Saúde (BVS - http://decs.bvs.br), utilizando-se as palavras-chave: "assistência farmacêutica", "programas estratégicos" e "medicamentos no Sistema Único de Saúde”. Consultou-se também a página da Secretaria de Atenção em Saúde (http://dtr2001.saude.gov.br/sas) na qual foi possível obter as portarias da própria Secretaria de Atenção em Saúde, do Gabinete do Ministro, do Departamento de Regulação, Avaliação e Controle de Sistemas. Nesse sítio, também foi possível consultar portarias conjuntas entre a 
Secretaria Executiva e Secretaria de Atenção em Saúde, portarias conjuntas da Secretaria de Atenção em Saúde e da Secretaria de Políticas de Saúde e acessar algumas consultas públicas. Outras importantes fontes de consulta foram as páginas de Internet da Secretaria de Vigilância em Saúde (http://portal.saude.gov.br/portal/ svs/area.cfm?id_area=418) e do Programa Nacional de DST e AIDS (http:/ / www.aids.gov.br/data/ Pages/LUMISFDF29F77PTBRIE.htm), bem como a informação disponibilizada pelo Departamento de Assistência Farmacêutica e Insumos Estratégicos da Secretaria de Ciência, Tecnologia e Insumos Estratégicos do Ministério da Saúde.

Foram considerados três critérios para identificação de um Programa de Assistência Farmacêutica: (1) Programas de Assistência Farmacêutica instituídos por Legislação específica até dezembro de 2005; (2) Programas de Assistência Farmacêutica cujo elenco encontrava-se presente em sítios oficiais do Ministério da Saúde até dezembro de 2005 e (3) Programas de Assistência Farmacêutica encaminhados, em agosto de 2004, pelo Departamento de Assistência Farmacêutica e Insumos Estratégicos por correio eletrônico.

\section{Construção do banco de dados}

Após identificar os Programas de Assistência Farmacêutica e seus respectivos elencos, foi construído um banco de dados contendo todos os medicamentos e produtos não terapêuticos encontrados nos programas de assistência farmacêutica e na RENAME, considerando suas respectivas nomenclaturas. Os medicamentos foram agregados pelo código ATC, segundo o Anatomical Therapeutic Chemical Classification System 13. Outras variáveis incluídas no banco foram: (1) forma farmacêutica (apresentação/concentração por dose); (2) unidade de apresentação da dose; e (3) via de administração, de acordo com o Anatomical Therapeutic Chemical Classification System 13, Ministério da Saúde (http:// portal.saude.gov.br/saude) e Thompson Healthcare Series/Micromedex (https://www.thom sonhc.com/hcs/librarian/PDFefaultActionId/ pf.LoginAction/ssl/true?ND_CPR=Login\&login. username_index_0=C7A0P7E2S\&login.pas sword_index_0+my816ju455).

\section{Definição dos medicamentos considerados prioritários para a ação do PROVEME}

Os medicamentos prioritários foram identificados segundo o critério presença, em qualquer apresentação, em, no mínimo, três Programas de Assistência Farmacêutica do Ministério da Saúde. Acredita-se que se três áreas do Ministério da
Saúde selecionam determinado fármaco, ele provavelmente responde a uma maior necessidade de saúde, e o monitoramento de sua qualidade é de importância para a Saúde Coletiva. Uma vez atendido a esse critério, foram considerados critérios adicionais, para hierarquização da importância dos medicamentos definidos como prioritários, sua presença no elenco da RENAME 2002 - que é a lista de medicamentos essenciais do Brasil - e sua indicação para, pelo menos, uma das vinte principais causas de DALY no Brasil, segundo documentos oficiais do Ministério da Saúde 14,15,16,17,18,19,20,21.

\section{Resultados}

Foram identificados, até dezembro de 2005, 13 Programas de Assistência Farmacêutica do Ministério da Saúde, contendo um total de 960 fármacos, produtos auxiliares não terapêuticos, vacinas e imunobiológicos. Cada fármaco gerou um registro no banco de dados, mas 67 (6,9\%) desses registros, relativos a medicamentos distribuídos pelos programas de DST e AIDS e controle de endemias, não continham informações completas para a análise. Entre os medicamentos antiretrovirais, $77,1 \%$ não puderam ser analisados pela ausência de informações (especialmente forma farmacêutica, dose e apresentação). Dos medicamentos listados para tratamento de DST, nenhum possuía informação completa.

Restaram 893 registros válidos para análise, correspondentes a 431 produtos, sendo 59 $(13,7 \%)$ vacinas, produtos imunobiológicos e outros produtos auxiliares não terapêuticos. Os 372 fármacos encontravam-se em 755 apresentações farmacêuticas, sendo 353 (81,9\%) monofármacos e $19(4,4 \%)$ medicamentos com mais de um princípio ativo, que se distribuíam, por vezes, em mais de um programa de assistência farmacêutica, conforme a Tabela 1.

O Programa de Medicamentos Excepcionais foi o que apresentou o maior número de fármacos, ou seja, $27,7 \%$ do total e $31,6 \%$ das apresentações farmacêuticas encontradas. Deve-se ressaltar que os Programas Assistência Farmacêutica da Saúde da Família; Saúde da Mulher e Assistência Farmacêutica para Hipertensão Arterial e Diabetes Mellitus foram considerados independentes do Programa de Assistência Farmacêutica na Atenção Básica, pois a Portaria GM $n^{o}$. 2.084 de 26 de outubro de 200516 não revoga as portarias que os instituíram.

Os 893 produtos foram classificados em 449 diferentes códigos ATC. Os grupos anatômicos mais freqüentes, no lo nível ATC, foram os (J) antiinfecciosos gerais para uso sistêmico $(21,1 \%)$, 
Distribuição de fármacos e apresentações farmacêuticas, segundo a Relação Nacional de Medicamentos Essenciais, 2002, e os Programas de Assistência Farmacêutica do Ministério da Saúde identificados até dezembro de 2005. Brasil, 2006.

\begin{tabular}{|c|c|c|c|c|}
\hline \multirow[t]{2}{*}{ Programas da Assistência Farmacêutica do Ministério da Saúde } & \multicolumn{2}{|c|}{ Fármacos } & \multicolumn{2}{|c|}{ Apresentações } \\
\hline & $\mathbf{n}$ & $\%$ & $\mathbf{n}$ & $\%$ \\
\hline 1. Programa de Medicamentos Excepcionais & 103 & 27,7 & 239 & 31,6 \\
\hline 2. Programa Farmácia Popular do Brasil & 72 & 19,3 & 102 & 13,5 \\
\hline 3. Programa Assistência Farmacêutica na Atenção Básica & 42 & 11,3 & 63 & 8,3 \\
\hline 4. Programa Nacional de Imunizações & 32 & 8,6 & 56 & 7,4 \\
\hline 5. Programa Estratégico de Endemias * & 30 & 8,0 & 45 & 6,0 \\
\hline 6. Programa de Saúde Mental & 19 & 5,1 & 39 & 5,2 \\
\hline 7. Programa Nacional de DST/AIDS & 24 & 6,4 & 24 & 3,2 \\
\hline 8. Programa Saúde da Família & 20 & 5,4 & 23 & 3,0 \\
\hline 9. Programa Estratégico Hemoderivados & 6 & 1,6 & 15 & 2,0 \\
\hline 10. Programa Estratégico Saúde da Mulher & 5 & 1,3 & 5 & 0,6 \\
\hline 11. Programa Nacional de Assistência Farmacêutica para Hipertensão Arterial e Diabetes Mellitus & 6 & 1,6 & 6 & 0,8 \\
\hline 12. Diagnóstico Imunológico & 1 & 0,2 & 1 & 0,1 \\
\hline 13. Programa de Distribuição de Insulina & 1 & 0,2 & 1 & 0,1 \\
\hline
\end{tabular}

* Inclui o elenco de medicamentos utilizados para tratamento das seguintes doenças: doença de chagas, esquistossomose, filariose, hanseníase, leishmanioses, malária, peste, tracoma e tuberculose.

(N) sistema nervoso central, (13,1\%), (L) agentes antineoplásicos e imunomoduladores $(11,3 \%)$, (C) sistema cardiovascular e (A) trato alimentar e metabolismo, ambos com $(7,8 \%)$ e (B) sangue e órgãos hematopoiéticos $(6,7 \%)$, sendo que, para dois registros $(0,4 \%)$, não foi encontrado código ATC correspondente.

No 2o nível da classificação ATC, verificou-se que os grupos terapêuticos principais em maior percentual foram: (J01) antibacterianos para uso sistêmico (6,9\%), (L01) agentes antineoplásicos (5,5\%), (J07) vacinas $(4,7 \%)$ e os (J05) antivirais para uso sistêmico (4\%) (Tabela 2).

No 3o nível ATC (Tabela 3), observou-se que os subgrupos terapêuticos/farmacológicos de maior freqüência foram: (J05A) antivirais de atividade direta (4\%), (V03A) demais produtos de uso terapêutico $(3,3 \%)$, $(\mathrm{C} 10 \mathrm{~A})$ agentes modificadores do perfil lipídico - monofármacos $(2,4 \%),(\mathrm{J} 07 \mathrm{~B})$ vacinas virais $(2,4 \%),(\mathrm{J} 07 \mathrm{~A})$ vacinas bacterianas $(2,2 \%)$, (L03A) imunomoduladores e citoquinas $(2,2 \%)$ e os (N03A) antiepilético $(2,2 \%)$.

No $4^{\circ}$ nível ATC (Tabela 4), os subgrupos terapêuticos/farmacológicos/químicos que apresentaram as maiores freqüências foram: (V03AB) antídotos (2,5\%), (B05XA) soluções eletrolíticas $(1,3 \%),(\mathrm{C} 10 \mathrm{AB})$ fibratos $(1,3 \%),(\mathrm{J} 05 \mathrm{AE})$ inibidores da protease (1,3\%), (J05AF) nucleosídeo e nucleotídeo inibidores da transcriptase reversa (1,3\%), (J06AA) anti-soros (1,3\%), (L03AB) inter- ferons (1,3\%), (L04AA) agente imunossupressor seletivo (1,3\%), (B02BD) fatores de coagulação sanguínea (1,1\%), (C10AA) inibidores da HMGCoA redutase $(1,1 \%)$ e (J06BB) imunoglobulinas específicas $(1,1 \%)$.

Existem 28 fármacos presentes em, no mínimo, três Programas de Assistência Farmacêutica do Ministério da Saúde. Verificou-se que 26 deles fazem parte do elenco da RENAME, e 12 possuem indicação no tratamento das vinte principais doenças, considerando os anos de vida perdidos ajustados por incapacidade (DALYs - Tabela 5).

\section{Discussão}

Deve-se ressaltar a dificuldade de identificação clara dos Programas de Assistência Farmacêutica e de seus respectivos elencos farmacêuticos no período do estudo. As sucessivas normas, até 2005, não se mostraram esclarecedoras quanto à revogação de suas antecessoras e à extinção ou não dos Programas de Assistência Farmacêutica incorporados na Portaria GM $n^{o} .2 .084$ de 26 de outubro de 2005 16. Há tentativas atuais de melhor definir os Programas de Assistência Farmacêutica 22 , no entanto, apesar de melhor organização do instrumento legal, inegável ajuda para o gestor, este ainda apresenta falta de definição no que tange aos elencos e à informação disponibilizada. 
Distribuição, segundo 2o nível da Anatomical Therapeutic Chemical Classification (ATC), dos fármacos presentes na Relação Nacional de Medicamentos Essenciais e nos Programas de Assistência Farmacêutica do Ministério da Saúde identificados até dezembro de 2005. Brasil, 2006

\begin{tabular}{|c|c|c|}
\hline 2o nível da ATC & $\mathbf{n}$ & $\%$ \\
\hline J01 - antibacterianos para uso sistêmico & 31 & 6,9 \\
\hline L01 - agentes antineoplásicos & 25 & 5,5 \\
\hline J07 - vacinas & 21 & 4,7 \\
\hline J05 - antivirais para uso sistêmico & 18 & 4,0 \\
\hline G03 - hormônios sexuais e moduladores do sistema reprodutor & 15 & 3,4 \\
\hline V03 - demais produtos de uso terapêutico & 15 & 3,4 \\
\hline B05 - substitutos do sangue e soluções para perfusão & 14 & 3,1 \\
\hline J06 - anti-soros e imunoglobulinas & 12 & 2,7 \\
\hline P01 - antiprotozoários & 12 & 2,7 \\
\hline C10 - agentes modificadores do perfil lipídico & 11 & 2,4 \\
\hline N05 - psicolépticos & 11 & 2,4 \\
\hline R03 - fármacos para desobstrução das vias aéreas & 11 & 2,4 \\
\hline LO3 - imunoestimulantes & 10 & 2,2 \\
\hline N01 - anestésicos & 10 & 2,2 \\
\hline N03 - antiepiléticos & 10 & 2,2 \\
\hline S01 - oftalmológicos & 10 & 2,2 \\
\hline Outros & 213 & 47,2 \\
\hline Ignorado * & 2 & 0,4 \\
\hline Total & 451 & 100,0 \\
\hline
\end{tabular}

* Não foi possível a classificação de dois registros no código ATC

Tabela 3

Distribuição, segundo 3o nível da Anatomical Therapeutic Chemical Classification (ATC), dos fármacos presentes na Relação Nacional de Medicamentos Essenciais 2002 e nos Programas de Assistência Farmacêutica do Ministério da Saúde identificados até dezembro de 2005. Brasil, 2006.

\begin{tabular}{|c|c|c|}
\hline 3o nível da ATC & $\mathbf{n}$ & $\%$ \\
\hline J05A - antivirais de atividade direta & 18 & 4,0 \\
\hline V03A - demais produtos de uso terapêutico & 15 & 3,3 \\
\hline C10A - agentes modificadores do perfil lipídico - monofármacos & 11 & 2,4 \\
\hline J07B - vacinas virais & 11 & 2,4 \\
\hline J07A - vacinas bacterianas & 10 & 2,2 \\
\hline LO3A - imunomoduladores e citoquinas & 10 & 2,2 \\
\hline N03A - antiepilético & 10 & 2,2 \\
\hline L04A - agente imunosupressor & 9 & 2,0 \\
\hline N05A - antipsicótico & 9 & 2,0 \\
\hline J01C - antibacterianos betalactâmicos, penicilinas & 8 & 1,8 \\
\hline P01B - antimaláricos & 8 & 1,8 \\
\hline N04B - agentes dopaminérgicos & 7 & 1,6 \\
\hline Outros & 323 & 71,6 \\
\hline Ignorado * & 2 & 0,4 \\
\hline Total & 451 & 100,0 \\
\hline
\end{tabular}

* Não foi possível a classificação de dois registros no código ATC 
Distribuição, segundo 4o nível da Anatomical Therapeutic Chemical Classification (ATC), dos fármacos presentes na Relação Nacional de Medicamentos Essenciais 2002 e nos Programas de Assistência Farmacêutica do Ministério da Saúde identificados até dezembro de 2005. Brasil, 2006.

\begin{tabular}{|c|c|c|}
\hline 4o nível da ATC & $\mathbf{n}$ & $\%$ \\
\hline V03AB - antídotos & 11 & 2,5 \\
\hline B05XA - soluções eletrolíticas & 6 & 1,3 \\
\hline C10AB - fibratos & 6 & 1,3 \\
\hline J05AE - inibidores da protease & 6 & 1,3 \\
\hline J05AF - nucleosídeos e nucleotídeos inibidores da transcriptase reversa & 6 & 1,3 \\
\hline J06AA - anti-soros & 6 & 1,3 \\
\hline L03AB - interferons & 6 & 1,3 \\
\hline L04AA - agentes imunossupressores seletivos & 6 & 1,3 \\
\hline B02BD - fatores de coagulação sanguínea & 5 & 1,1 \\
\hline C10AA - inibidores da HMG-CoA redutase & 5 & 1,1 \\
\hline J06BB - imunoglobulinas específicas & 5 & 1,1 \\
\hline N01BB - amidas & 5 & 1,1 \\
\hline H02AB - glucocorticóides & 4 & 0,9 \\
\hline J01CE - penicilinas sensíveis à beta lactamase & 4 & 0,9 \\
\hline J01FA - macrolídeos & 4 & 0,9 \\
\hline J05AB - nucleosídeos e nucleotídeos exceto inibidores de transcriptase reversa & 4 & 0,9 \\
\hline L01AA - análogos de mostardas nitrogenadas & 4 & 0,9 \\
\hline Outros & 356 & 79 \\
\hline Ignorado * & 2 & 0,5 \\
\hline Total & 451 & 100,0 \\
\hline
\end{tabular}

* Não foi possível a classificação de dois registros no código ATC.

Durante a identificação dos Programas de Assistência Farmacêutica do Ministério da Saúde, observou-se que os medicamentos são apresentados pelos distintos programas de assistência farmacêutica e pela RENAME-2002 de forma pouco homogênea, quanto à nomenclatura e quanto às respectivas formas farmacêuticas. Por serem a base para as demais ações relativas à Assistência Farmacêutica e para o Uso Racional dos Medicamentos, a insuficiente especificação do medicamento selecionado pode comprometer diretrizes da Política Nacional de Medicamentos: gestão da Assistência Farmacêutica e prescrição racional.

A Organização Mundial da Saúde (OMS) e especialistas da área da gestão da qualidade enfatizam a padronização dos insumos, dos medicamentos e dos protocolos de tratamento, diante da necessidade de se atender às demandas geradas pelo paciente e pelo gerenciamento de custos 23 . A não especificação de determinadas características, como a forma química do princípio ativo ou a apresentação completa, pode induzir a erros que implicam em risco à saúde e/ou dificultam a tomada de decisões.
Foram encontradas, nos protocolos e diretrizes oficiais do Ministério da Saúde, indicações terapêuticas nas principais causas de DALY para doze dos 28 fármacos presentes em, pelo menos, três Programas de Assistência Farmacêutica do Ministério da Saúde e definidos como prioritários para a ação do PROVEME. São eles: ácido acetilsalicílico, benzoato de benzila, captopril, ciprofloxacino, glibenclamida, metformina, metronidazol, prednisona, propranolol, salbutamol sulfametoxazol associado com trimetoprima e sulfato ferroso. Não foi possível correlacionar os fármacos para as seguintes causas principais de DALY: doenças cerebrovasculares (infarto cerebral, acidente vascular cerebral); doença pulmonar obstrutiva crônica; transtornos mentais/ comportamentais - uso de álcool; outras desordens endócrinas/metabólicas; cirrose hepática e aborto. Nessas situações, a causa principal de DALY não está explicitamente vinculada a um tratamento ou conjunto de fármacos, mas a um conjunto de condutas e medidas terapêuticas.

Os Programas de Assistência Farmacêutica do Ministério da Saúde foram criados no intuito de melhorar o acesso aos medicamentos pela 
Fármacos selecionados para o monitoramento pelo Programa Nacional de Verificação da Qualidade de Medicamentos e critérios utilizados para sua seleção. Brasil, 2006

\begin{tabular}{|c|c|c|c|}
\hline Nome do fármaco & $\begin{array}{c}\text { Programas de Assistência Farmacêutica } \\
\text { do Ministério da Saúde identificados } \\
\text { até dezembro de } 2005\end{array}$ & $\begin{array}{c}\text { RENAME } \\
2002\end{array}$ & $\begin{array}{c}\text { Carga } \\
\text { de } \\
\text { doença }\end{array}$ \\
\hline Acetato de medroxiprogesterona & PSMU; PAB e PFPOB & - & - \\
\hline Acido acetilsalicílico & PAB; PSF e PFPOB & Sim & Sim \\
\hline Albendazol & PAB; PDST/AIDS e PFPOB & $\operatorname{Sim}$ & - \\
\hline Amoxicilina & PAB; PSF e PFPOB & Sim & - \\
\hline Benzilpenicilina benzatina & PAB; PSF e PFPOB & Sim & - \\
\hline Benzoato de benzila & PAB; PSF e PFPOB & Sim & Sim \\
\hline Biperideno & PME; PSME e PFPOB & $\operatorname{Sim}$ & - \\
\hline Captopril & PAB; PHD; PSF e PFPOB & $\operatorname{Sim}$ & $\operatorname{Sim}$ \\
\hline Ciprofloxacino & PME; PDST/AIDS E PFPOB & Sim & Sim \\
\hline Dexametasona & PAB; PSF e PFPOB & Sim & - \\
\hline Enantato de noretisterona + valerato de Estradiol & PSMU; PAB e PFPOB & - & - \\
\hline Etinilestradiol + levonorgestrel & PSMU; PAB e PFPOB & Sim & - \\
\hline Glibenclamida & PAB; PHD; PSF e PFPOB & Sim & Sim \\
\hline Hidroclorotiazida & PAB; PHD; PSF e PFPOB & $\operatorname{Sim}$ & - \\
\hline Levodopa + carbidopa & PME; PSME e PFPOB & Sim & - \\
\hline Levonorgestrel & PSMU; PAB e PFPOB & Sim & - \\
\hline Mebendazol & PAB; PSF e PFPOB & Sim & - \\
\hline Metformina & PAB; PHD e PFPOB & $\operatorname{Sim}$ & $\operatorname{Sim}$ \\
\hline Metildopa & PAB; PSF e PFPOB & Sim & - \\
\hline Metronidazol & PAB; PSF e PFPOB & Sim & Sim \\
\hline Neomicina + bacitracina & PAB; PSF e PFPOB & Sim & - \\
\hline Noretisterona & PSMU; PAB e PFPOB & $\operatorname{Sim}$ & - \\
\hline Paracetamol & PAB; PSF e PFPOB & $\operatorname{Sim}$ & - \\
\hline Prednisona & PEND; PAB e PFPOB & Sim & $\operatorname{Sim}$ \\
\hline Propranolol & PAB; PHD; PSF e PFPOB & $\operatorname{Sim}$ & $\operatorname{Sim}$ \\
\hline Salbutamol & PAB; PME; PSF e PFPOB & Sim & Sim \\
\hline Sulfametoxazol associado com trimetoprima & PEND; PAB; PDST/AIDS e PFPOB & Sim & Sim \\
\hline Sulfato ferroso & PAB; PSF e PFPOB & Sim & Sim \\
\hline
\end{tabular}

RENAME: Relação Nacional de Medicamentos Essenciais; PSMU: Programa Estratégico Saúde da Mulher; PAB: Programa Assistência Farmacêutica na Atenção Básica; PFPOB: Programa Farmácia Popular do Brasil; PSF: Programa Saúde da Família; PDST/AIDS: Programa Nacional de DST/AIDS; PME: Programa de Medicamentos Excepcionais; PSME: Programa de Saúde Mental; PHD: Programa Nacional de Assistência Farmacêutica para Hipertensão Arterial e Diabetes Mellitus; PEND: Programa Estratégico de Endemias.

população. Em 2005, cada programa apresentava um elenco próprio de medicamentos. Desses elencos, faziam parte medicamentos presentes na RENAME-2002 e outros que, por suas particularidades, tornavam-se necessários para o êxito do programa. Analisando os fármacos, em seu quinto nível de classificação ATC, observouse que cerca de $21 \%$ (93) não faziam parte da RENAME-2002. Uma vez que a RENAME contém apenas medicamentos considerados de primeira e segunda linha, para as enfermidades mais prevalentes no país, esse percentual foi, na verdade, bastante positivo.
O transtorno depressivo é a quarta causa de DALY na população brasileira como um todo e a primeira entre as mulheres 1 . O Relatório Mundial da Saúde da OMS recomenda a disponibilização de antidepressivos, considerados essenciais, em todos os níveis de cuidados de saúde 24,25. No Brasil, a Pesquisa Mundial em Saúde referiu que $26 \%$ dos entrevistados relataram grau grave de problema relativo ao estado de ânimo; 19,3\% já tiveram confirmado diagnóstico para depressão 26 . Paradoxalmente, não foi identificada a presença de antidepressivo no elenco mínimo da Assistência Farmacêutica na Atenção Básica 16. 
Vale ressaltar que dois fármacos, medroxiprogesterona e noretisterona associado ao estradiol, que não se encontravam elencados na RENAME-2002, foram incorporados na revisão da RENAME-2006 27.

Pode-se considerar que os 28 fármacos identificados no estudo são prioritários não apenas para o PROVEME, mas para a Política Nacional de Medicamentos como um todo, sendo necessária a integração entre os diferentes órgãos do Ministério da Saúde que têm como objeto de atuação os medicamentos.

O presente estudo desenvolveu uma metodologia que se mostrou adequada para definir prioridades de ação para o PROVEME. Essa metodologia, no entanto, tem como limitação o fato de não considerar o consumo absoluto dos medicamentos como critério para definição de prioridades. Metodologias complementares, utilizando arcabouço conceitual dos estudos de utilização de medicamentos, podem contribuir para corroborar os resultados aqui apresentados e adicionar novas informações. Esses estudos estão em fase final de desenvolvimento.

\section{Considerações finais}

Tendo em vista os resultados deste estudo, sugerem-se algumas ações à ANVISA e ao Departamento de Assistência Farmacêutica e Insumos Estratégicos da Secretaria de Ciência, Tecnologia e Insumos Estratégicos do Ministério da Saúde.

À ANVISA, que o PROVEME considere, no monitoramento desses 28 fármacos, todos os produtos existentes no mercado e não apenas uma amostragem dos laboratórios produtores. Pode-se supor que esses fármacos estejam sendo largamente utilizados, independentemente da compra pelo Ministério da Saúde ou pelas secretarias estaduais e municipais de saúde.

Considerando a importância desses fármacos para a Saúde Coletiva e para a Política Nacional de Medicamentos, recomenda-se uma ação mais ampla que inclua, além do monitoramento da qualidade laboratorial, a avaliação de todas as etapas de sua cadeia de medicamentos. Assim, uma ação transversal deveria considerar, dentre outras: a inspeção do parque produtivo; a revisão de registros desses medicamentos; a análise e padronização da informação a ser veiculada para os profissionais e para o consumidor, na rotulagem e na propaganda, e investimento no uso racional desses fármacos. Ações essas que exigem um esforço de integração de diferentes setores dentro da própria ANVISA e integração dos componentes do Sistema Nacional de Vigilância Sanitária para que se possa atuar em toda a cadeia desses medicamentos, de sua produção à sua comercialização. Dessa forma, parece ser mais factível cumprir o objetivo da Política Nacional de Medicamentos, garantindo o acesso aos fármacos necessários e com qualidade.

Recomenda-se ao Departamento de Assistência Farmacêutica e Insumos Estratégicos a padronização das informações sobre medicamentos na RENAME e nos programas de Assistência Farmacêutica. Além disso, é necessária a sistematização dos elencos dos programas, considerando as informações completas sobre os fármacos e suas respectivas apresentações farmacêuticas, de forma a serem facilmente identificados. Essa é uma importante ação educativa, não apenas para os profissionais de saúde, mas também para a população. Conforma ainda às recentes orientações sobre Assistência Farmacêutica no país, formalizadas nas Portaria GM $n^{o} .204$ de 29 de janeiro de 200722 e Portaria GM $n^{\circ} .698$ de 30 de março de $2006{ }^{28}$, de garantir uma Assistência Farmacêutica voltada não apenas para o fornecimento de medicamentos, mas para a integralidade das ações no âmbito do SUS.

Tendo ainda em vista que a revisão dos elencos dos programas é periódica, como também é a revisão da RENAME 27, espera-se que o monitoramento proposto neste trabalho possa ser empregado de forma também periódica, mantendo atualizada a lista de medicamentos prioritários para o país para as ações pertinentes da ANVISA e do Ministério da Saúde. 


\section{Resumo}

A Política Nacional de Medicamentos tem como importante diretriz a qualidade dos medicamentos oferecidos à população. Objetivou-se definir prioridades para análise pelo Programa Nacional de Verificação da Qualidade de Medicamentos. Como critério, utilizou-se a presença do medicamento em, no mínimo, três Programas de Assistência Farmacêutica do Ministério da Saúde. Critérios adicionais foram a presença na Relação Nacional de Medicamentos Essenciais de 2002 (RENAME) e a indicação para as vinte principais causas de Anos de Vida Perdidos Ajustados por Incapacidade (DALY). Informações do Ministério da Saúde e legislação foram fontes da pesquisa. Classificaram-se os medicamentos segundo $o$ Anatomical Therapeutic Chemical Classification System (ATC) da OMS. Nos 13 programas de assistência farmacêutica, existiam 893 produtos classificados em 449 diferentes códigos ATC. Foram considerados prioritários 28 fármacos, 26 constantes na RENAME e 12 indicados nas causas de DALY. Recomenda-se, à Agência Nacional de Vigilância Sanitária e à Secretaria de Ciência, Tecnologia e Insumos Estratégicos, estabelecer estratégia integrada para garantia de qualidade integral desses medicamentos, abrangendo qualidade laboratorial, registro, boas práticas de fabricação e informações para profissionais de saúde e população.

Qualidade dos Medicamentos; Política Nacional de Medicamentos; Vigilância Sanitária

\section{Colaboradores}

D. M. Pontes Junior colaborou na pesquisa bibliográfica, coleta de dados, construção do banco de dados, análise dos dados, discussão, recomendações e redação do artigo. V. L. E. Pepe contribuiu na construção, revisão e análise do banco de dados, discussão, recomendações e revisão do texto final. C. G. S. Osorio-de-Castro participou na construção e revisão do banco de dados, redação e revisão do texto final. E. P. Massena contribuiu na coleta de dados (em programas e legislações), elaboração e atualização do banco de dados e discussão de alguns dados. M. C. Portela participou na redação e revisão do texto final. R. S. Silva colaborou na análise estatística dos dados e discussão dos resultados. M. C. Miranda contribuiu na revisão do texto final.

\section{Agradecimentos}

À Agência Nacional de Vigilância Sanitária (ANVISA), que financiou essa pesquisa por meio do Convênio Escola Nacional de Saúde Pública Sergio Arouca, Fundação Oswaldo Cruz (ENSP/FIOCRUZ/ANVISA): Projeto de Pesquisa e Desenvolvimento Institucional em Vigilância Sanitária. Ao Departamento de Assistência Farmacêutica e Insumos Estratégicos, Secretaria de Ciência, Tecnologia e Insumos Estratégicos, Ministério da Saúde, em especial, à Ana Márcia Messeder, pelas informações disponibilizadas e a ajuda prestada na resolução de dúvidas que surgiram durante a pesquisa. Ao aluno, Leonardo Lima Cymerman, do Programa de Vocação Científica (PROVOC) da Escola Politécnica de Saúde Joaquim Venâncio, FIOCRUZ, que nos ajudou durante a etapa de coleta de dados.

\section{Referências}

1. Gadelha AMJ, Leite IC, Valente JG, Schramm JMA, Portela MC, Campos MR, et al. Projeto carga de doenças [CD-ROM]. Rio de Janeiro: Fundação Oswaldo Cruz; 2002.

2. Carmo EH, Barreto ML, Silva Junior JB. Mudanças nos padrões de morbimortalidade da população brasileira: os desafios para um novo século. Epidemiol Serv Saúde 2003; 12:63-75.

3. Ministério da Saúde. Saúde Brasil 2005: uma análise da situação de saúde no Brasil. Brasília: Ministério da Saúde; 2005. (Série C. Projetos, Programas e Relatórios).

4. Brasil. Portaria GM/MS no. 3.916 de 30 de outubro de 1998. Aprova a Política Nacional de Medicamentos. Diário Oficial da União 1998; 10 nov.
5. Beltrane A. Aplicação do acesso a medicamentos de alto custo: uma análise da política brasileira [Dissertação de Mestrado]. Rio de Janeiro: Instituto de Medicina Social, Universidade do Estado do Rio de Janeiro; 2002.

6. Brasil. Resolução CNS no ${ }^{\circ} .338$ de 6 de maio de 2004. Aprova a Política Nacional de Assistência Farmacêutica. Diário Oficial da União 2004; 20 mai.

7. Ministério da Saúde. Política Federal de Assistência Farmacêutica 1990 a 2002. Brasília: Ministério da Saúde; 2002. (Série B. Textos Básicos de Saúde). 
8. Messeder AM, Osorio-de-Castro CGS, Luiza VL. Mandados judiciais como ferramenta para garantia do acesso a medicamentos no setor público: a experiência do Estado do Rio de Janeiro, Brasil. Cad Saúde Pública 2005; 21:525-34.

9. Bermudez JAZ, Possas CA. Análisis crítico de la política de medicamentos en el Brasil. Bol Oficina Sanit Panam 1995; 119:270-7.

10. Jaramillo NM, Ivama AM, Barbano DBA, Santos MRC, Luiza VL. Avaliação da assistência farmacêutica no Brasil: estrutura, processos e resultados. Brasília: Organização Pan-Americana da Saúde/ Ministério da Saúde; 2005. (Série Técnica. Medicamentos e Outros Insumos Essenciais para a Saúde).

11. Corrêa JCV. Qualidade dos medicamentos comercializados no Brasil segundo dados do Instituto Nacional de Controle de Qualidade em Saúde e do Instituto Adolfo Lutz [Dissertação de Mestrado]. São Paulo: Universidade de São Paulo; 2003.

12. Agência Nacional de Vigilância Sanitária. Diretrizes do Programa Nacional de Verificação da Qualidade de Medicamentos - PROVEME. http://www. anvisa.gov.br/inspecao/fiscalizacao/proveme/ manual_proveme.pdf (acessado em 28/Jun/2006).

13. WHO Collaborating Centre for Drugs Statistics Methodolosy. Anatomical Therapeutic Chemical Classifacation (ATC Code). http://www.whocc.no/ atcddd (acessado em 06/Jun/2006).

14. Wannmacher L, Bermudez JAZ, Osorio-de-Castro CGS, Reis ALA, Mosegui GBG, Gervásio GR, et al. Elaboração do formulário terapêutico nacional. In: VI Congresso Brasileiro de Saúde Coletiva 2000, livro de resumos. Rio de Janeiro: ABRASCO/Fundação Oswaldo Cruz; 2000. p. 13.

15. Departamento de Sistemas e Redes Assistenciais, Secretaria de Assistência à Saúde, Ministério da Saúde. Protocolos clínicos e diretrizes terapêuticas: medicamentos excepcionais. Brasília: Ministério da Saúde; 2002. (Série A. Normas e Manuais Técnicos).

16. Brasil. Portaria GM n ${ }^{\circ} .2 .084$ de 26 de outubro de 2005. Estabelece os mecanismos e as responsabilidades para o financiamento da Assistência Farmacêutica na Atenção Básica e dá outras providências. Diário Oficial da União 2005; 28 out.

17. Brasil. Portaria GM n ${ }^{\circ} .1 .318$ de 23 de julho de 2002. Inclui medicamentos na tabela de medicamentos excepcionais. Brasília: Diário Oficial da União 2002; 24 jul.
18. Brasil. Portaria SAS/MS no. 921 de 25 de novembro de 2002. Inclui medicamentos na tabela de medicamentos excepcionais. Diário Oficial da União 2002; 25 nov.

19. Brasil. Portaria SAS/MS no. 203 de 19 de abril de 2005. Incluir, na tabela de procedimentos especiais do Sistema de Informações Hospitalar do Sistema Único de Saúde - SIH/SUS, medicamentos excepcionais. Diário Oficial da União 2005; 20 abr.

20. Agência Nacional de Vigilância Sanitária. Fundamentos farmacológico-clínicos dos medicamentos de uso corrente. http://www.anvisa.gov.br/divul ga/public/livro_eletronico/INDEX.HTM (acessado em 10/Dez/2005).

21. Organização Mundial da Saúde. Classificação estatística internacional de doenças e problemas relacionados à saúde, 10a revisão. São Paulo: Centro Colaborador da OMS para a Classificação de Doenças em Português; 1995.

22. Brasil. Portaria GM nº. 204 de 29 de janeiro de 2007. Regulamenta o financiamento e a transferência dos recursos federais para as ações e os serviços de saúde, na forma de blocos de financiamento, com o respectivo monitoramento e controle. Diário Oficial da União 2007; 31 jan.

23. Luiza VL, Osorio-de-Castro CGS, Nunes JM. Aquisição de medicamentos no setor público: o binômio qualidade-custo. Cad Saúde Pública 1999; 15:769-76.

24. Wannmacher L. Depressão maior: da descoberta à solução. In: Uso racional de medicamentos: temas selecionados. http://www.opas.org.br/ medicamentos/\%docs/HSE_URM_DEP_0404.pdf (acessado em 26/Mai/2006).

25. The World Health Report 2001. Mental health: new understanding, new hope. http://www.who. int/whr/2001/en/index.html (acessado em 26/ Mai/2006)

26. Szwarcwald CL, Viacava F, Vasconcellos MTL, Leal MC, Azevedo LO, Queiroz RSB, et al. Pesquisa mundial de saúde - 2003: o Brasil em números. RADIS Comunicação em Saúde 2004; 1:14-33.

27. Brasil. Portaria GM n ${ }^{\circ} .2 .475$, de 13 de outubro de 2006. Aprova a 4o edição da Relação Nacional de Medicamentos Essências (RENAME). Diário Oficial da União 2006; 18 out.

28. Brasil. Portaria GM no. 698, de 30 de março de 2006. Define que o custeio das ações de saúde é de responsabilidade das três esferas de gestão do SUS, observado o disposto na Constituição Federal e na Lei Orgânica do SUS. Diário Oficial da União 2006; 3 abr.

Recebido em 04/Mai/2007

Versão final reapresentada em 30/Jan/2008 Aprovado em 12/Fev/2008 